\title{
ABSTRACTS OF CURRENT LITERATURE
}

\section{THE AMERICAN JOURNAL OF EMERGENCY MEDICINE}

\section{Medical Consultations and the Sharing of Medical Images Involving Spinal Injury Over Mobile Phone Networks}

This study aimed to evaluate the efficacy of mobile phone networks for remote medical consultations. This experimental study compared the use of mobile phone radiology consultation to hospital workstation radiology consultation for spinal injury patients in the Czech Republic.

Over a 1-year period, 17 cases were included based on the following criteria: 1) aged 18 to 90 years; 2) resident in neurosurgery on duty; and 3) patient with a potentially unstable spinal injury. For these cases, images were sent to both a hospital workstation using PACS (Picture Archiving and Communication System) and a mobile phone in JPEG format at a resolution of $200 \times 200$ pixels. Experienced neurosurgeons evaluated the images on the mobile phone device while neurosurgical residents received images on a hospital workstation. Total consultation time, accuracy of diagnosis, and image quality were the measured outcomes for this study.

There was full agreement for diagnosis and management for each case; however, the mobile phone consultation required approximately 8 minutes longer and more image adjustment (ie, zooming, rotating, shifting) than standard hospital workstation computers. The mobile phone image was determined to be sufficient for accurate diagnosis; however, there were no stated objective criteria for quality. Limitations of the study included sample size, inadequate description of mobile phone image quality, applicability to other injury patterns, and varying international mobile phone network speeds. Although the study has obvious limitations, the authors suggested that as mobile phone technology progresses, it will become an increasingly integral component of utilizing remote medical expertise.

(Am J Emerg Med. 2011 [Epub Ahead of print]) M Filip, $\mathrm{P}$ Linzer, F Samal, et al.

Prepared by Matthew Stewart, MD, University of Utah Emergency Medicine Resident, Salt Lake City, UT, USA.

\section{WESTERN JOURNAL OF EMERGENCY MEDICINE}

\section{Mass Casualty Incident Response and Aeromedical Evacuation in Antarctica}

This article summaries the current contingency operations and resources for a mass casualty incident (MCI) in Antarctica. The authors cited surveyed literature and past incidents to describe the current state of medical preparedness. They also noted the increased number of personnel in Antarctica owing to tourism and research, noting a presence of more than 30 nations, 80 research stations, and 10000 tourists during the summer months. These numbers dwindle to 1000 people in the winter.

The authors noted several events that demonstrate the potential for an MCI. Two major earthquakes occurred in 1998 and 2004 that reached a peak amplitude of 8.1 on the Richter scale. Airlines have had crashes, for example, flight 901 from New Zealand in 1979, killing all of the passengers; and ships have also been stranded recently, including the MS Explorer in 2007. Researchers have also had problems with laboratory fires in 2001 in the United Kingdom and in 2008 in Russia.

Search and rescue capabilities are divided into 5 regions including New Zealand, Australia, South Africa, Argentina, and Chile. There is no common contingency plan for an MCI, and a region would respond to a nearby distress signal or GPS signal from an emergency radio beacon. The authors suggest further collaboration to coordinate and prepare for any future MCIs. Additionally, current resources include possible staging at McMurdo Hospital, which has no surgical or critical care capability and is 2400 miles away from the nearest hospital in New Zealand. A response would most likely utilize military transportation, such as critical care air transport teams on C-130 or C-17 aircraft. Otherwise, mobile hospitals can be brought to an MCI site on Antarctica.

(West J Emerg Med. 2011;12:37-42) CN Mills, GH Mills. Prepared by Anil Menon, MD, UTMB/NASA Aerospace Medicine Fellow, Galveston, TX, USA.

\section{MORBIDITY AND MORTALITY WEEKLY REPORT}

\section{Nonfatal Sports and Recreation Heat Illness Treated in Hospital Emergency Departments-United States 2001-2009}

This report provided valuable information about the incidence and characteristics of heat illness related to sports and recreation. The Centers for Disease Control (CDC) analyzed data from the National Electronic Injury Surveillance System (NEISS) from 2001 to 2009 and found that approximately 5946 people were treated in a US Emergency Department (ED) each year for heat illness. The NEISS monitors consumer productrelated injuries treated in 100 US hospital EDs. Cases were excluded that were not precipitated by natural or environmental causes.

The incidence of heat illness was highest among males $(72.5 \%)$ and persons aged 15 to 19 years $(35.6 \%)$. The most common activities causing ED visits for heat illness were football $(24.5 \%)$ and exercise, identified as walking, jogging, 